\title{
FROM STRUCTURAL ANALYSIS TO OBSERVER-BASED RESIDUAL GENERATION FOR FAULT DETECTION
}

\author{
SEBASTIAN PRÖLL ${ }^{a, *}$, JAN LUNZE $^{b}$, FABIAN JARMOLOWITZ ${ }^{a}$ \\ ${ }^{a}$ Robert Bosch GmbH \\ Robert-Bosch-Campus 1, D-71272 Renningen, Germany \\ e-mail: sebastian.proell, fabian.jarmolowitz@de.bosch.com \\ ${ }^{b}$ Institute of Automation and Computer Control \\ Ruhr University Bochum, Universitätsstraße 150, D-44801 Bochum, Germany \\ e-mail: Lunzedatp.rub.de
}

\begin{abstract}
This paper combines methods for the structural analysis of bipartite graphs with observer-based residual generation. The analysis of bipartite structure graphs leads to over-determined subsets of equations within a system model, which make it possible to compute residuals for fault detection. In observer-based diagnosis, by contrast, an observability analysis finds observable subsystems, for which residuals can be generated by state observers. This paper reveals a fundamental relationship between these two graph-theoretic approaches to diagnosability analysis and shows that for linear systems the structurally over-determined set of model equations equals the output connected part of the system. Moreover, a condition is proved which allows us to verify structural observability of a system by means of the corresponding bipartite graph. An important consequence of this result is a comprehensive approach to fault detection systems, which starts with finding the over-determined part of a given system by means of a bipartite structure graph and continues with designing an observerbased residual generator for the fault-detectable subsystem found in the first step.
\end{abstract}

Keywords: fault diagnosis, structural analysis, observer-based diagnosis, diagnosability analysis.

\section{Introduction}

Fault detection is the task which refers to testing whether the input and output signals measured for a system $\Sigma$ are in consistency with the model of the faultless system (Fig. 1). It is well known that the information about the behavior of the system $\Sigma$ has to include redundancies in order to make fault diagnosis possible. Analysis methods have been elaborated to find such redundancies in the structure graph of the system $\Sigma$, which is a bipartite graph showing how the internal and external signals are combined by the model equations (Blanke et al., 2016). The analysis result is a set $\mathcal{C}^{+}$of over-determined equations that define the structurally diagnosable part of the system. From this set $\mathcal{C}^{+}$analytical redundancy relations (ARRs)

$$
\boldsymbol{r}(t)=\boldsymbol{g}(\boldsymbol{u}(t), \dot{\boldsymbol{u}}(t), \ldots, \boldsymbol{y}(t), \dot{\boldsymbol{y}}(t), \ldots)
$$

\footnotetext{
* Corresponding author
}

can be obtained to generate residuals, where, in general, the function $\boldsymbol{g}$ combines the measured input and output signals and their derivatives.

A system of equations has to be solved in order to implement a residual generator (1), which poses a serious problem in general. Ways how to deal with this problem have been proposed by Svärd and Nyberg (2010) as well as Lunze (2017). Even if an ARR is obtained, a direct practical application of (1) is generally impossible due to the derivatives that usually cannot be measured. Therefore, observer-based diagnostic methods have become a popular means for fault diagnosis (Ding, 2013). These methods reconstruct the internal state $\boldsymbol{x}(t)$ of the system $\Sigma$ in an asymptotic way and avoid the determination of the derivatives of the input and output signals. However, they can be applied only if the system is observable and, thus, makes it possible to reconstruct the internal variables for the measured pair $(\boldsymbol{u}(t), \boldsymbol{y}(t))$ of input and output signals. 


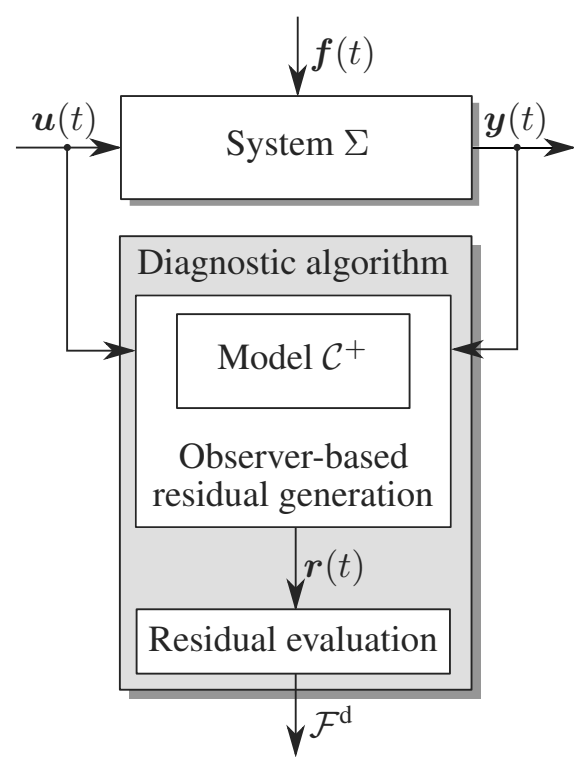

Fig. 1. Diagnosis of system $\Sigma$.

Structural analysis and observer-based fault diagnosis are two research topics which have been tackled completely separately in the past, with few exceptions like, e.g., the work of Bregon et al. (2014). For applications, however, it is necessary to find a way for applying observer-based techniques to those systems that have been shown to be structurally diagnosable. The aim of this paper is to uncover the relation between the mentioned research topics to make it possible to find observer-based fault detection and isolation algorithms for systems that have been proved to be structurally diagnosable. The paper shows that the structurally over-determined part $\mathcal{C}^{+}$of the model defines a structurally observable system if it is possible to find a suitable matching in the bipartite structure graph of the system (Theorem 4).

An important consequence of this new result is the possibility to first analyze a system $\Sigma$ by graphical means to find diagnosable subsystems and, second, to apply observer-based diagnostic methods to the observable subsystems found. Hence, a comprehensive manner of fault detection can be presented in Section 5. Moreover, this paper provides a theoretical basis for a comprehensive fault isolation method, which likewise combines structural analysis and observer-based diagnosis.

1.1. Literature. This paper builds upon the two lines of research mentioned above. The main ideas of structural analysis using bipartite graphs are explained in the monograph of Blanke et al. (2016). Structural analysis methods have become popular in the FDI community to analyze the diagnosability of continuous (Krysander, 2006; Krysander and Frisk, 2008) and hybrid systems (Pröll et al., 2015). Algorithms for determining over-determined sets of equations have been proposed by Armengol et al. (2009) and Frisk et al. (2012), with the latter also dealing with the causal interpretation of the model equations.

On the other hand, summaries of observer-based diagnostic methods can be found in the work of Ding (2013) and Isermann (2006). Geometric conditions for observer-based fault detection and isolation have been given by Massoumnia et al. (1989) or De Persis and Isidori (2001) for linear or nonlinear systems, respectively.

In the present paper, by contrast, graph-theoretic conditions for observability are investigated. The property of structural observability, which will be used to prove the main result, has been defined by Lin (1974) (for the dual controllability property) and the results along this line are summarised, for example, by Lunze (2013) and Reinschke (1988). Other criteria for state and input observability of linear state-space and differential-algebraic systems using directed graphs have been stated by Boukhobza et al. (2006; 2007). Structural observability has also been used for diagnosis in, e.g., the work of Commault and Dion (2007).

1.2. Structure of the paper. Section 2 states the problem of fault detection and Section 3 reviews known results on the structural analysis of the diagnosability and observability of linear systems. The notion of structural observability of a system is extended to structurally observable subsystems in Section 4. The main result shows the relation between diagnosability and observability as a basis for creating a thorough diagnostic method in Section 5. Section 6illustrates the results by an example.

\section{Diagnostic problem and its solution}

This paper considers linear time-invariant control systems

$$
\Sigma:\left\{\begin{array}{l}
\dot{\boldsymbol{x}}(t)=\boldsymbol{A} \boldsymbol{x}(t)+\boldsymbol{B} \boldsymbol{u}(t), \quad \boldsymbol{x}(0)=\boldsymbol{x}_{0}, \\
\boldsymbol{y}(t)=\boldsymbol{C} \boldsymbol{x}(t)
\end{array}\right.
$$

with known input $\boldsymbol{u}(\cdot) \in \mathbb{R}^{m}$, immeasurable state $\boldsymbol{x}(\cdot) \in$ $\mathbb{R}^{n}$, and known output $\boldsymbol{y}(\cdot) \in \mathbb{R}^{p}$. The matrices $\boldsymbol{A}, \boldsymbol{B}, \boldsymbol{C}$ have appropriate dimensions. The above model is also called a state-space model with outputs, or simply a statespace model. In such a model, the dynamics of each state variable $x_{i}(t)$ is declared by a first-order ordinary differential equation. Such a model is meant whenever one speaks of a system or a subsystem in the following.

The task is to detect faults $\boldsymbol{f}(\cdot) \in \mathbb{R}^{l}$ that appear as an additional term in the state or output equations, 


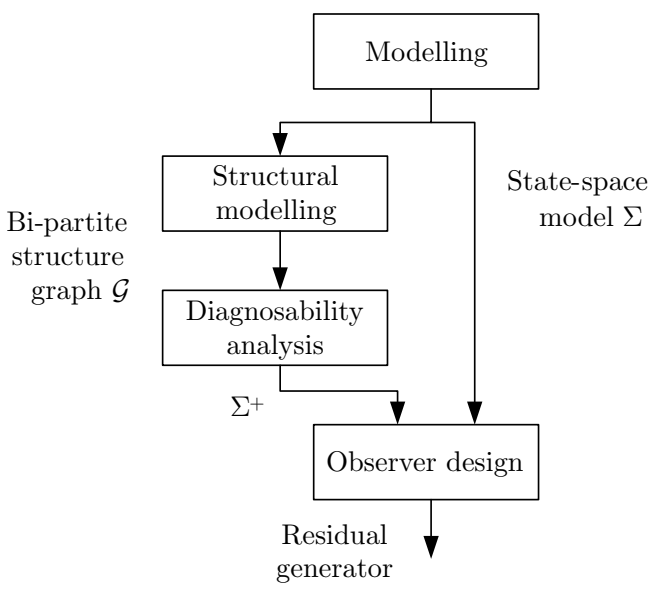

Fig. 2. Comprehensive way to create a fault diagnosis system.

respectively, and lead to the following model of the faulty system $\Sigma_{f}$ :

$$
\Sigma_{f}:\left\{\begin{array}{l}
\dot{\boldsymbol{x}}(t)=\boldsymbol{A} \boldsymbol{x}(t)+\boldsymbol{B} \boldsymbol{u}(t)+\boldsymbol{E}_{\mathrm{s}} \boldsymbol{f}(t), \\
\boldsymbol{x}(0)=\boldsymbol{x}_{0}, \\
\boldsymbol{y}(t)=\boldsymbol{C} \boldsymbol{x}(t)+\boldsymbol{E}_{\mathrm{o}} \boldsymbol{f}(t) .
\end{array}\right.
$$

Non-zero elements in the matrices $\boldsymbol{E}_{\mathrm{s}} \in \mathbb{R}^{n \times l}$ and $\boldsymbol{E}_{\mathrm{o}} \in \mathbb{R}^{p \times l}$ indicate which equations are affected by which fault. For fault detection, the diagnostic system should distinguish between the faultless case $(\boldsymbol{f}(t) \equiv \mathbf{0})$ and the faulty case $(\boldsymbol{f}(t) \neq \mathbf{0})$. This paper focusses on fault detection, which only needs the information about which model equation is affected by faults. All results can be extended to fault isolation if it is known how the system dynamics is affected by which fault.

This paper investigates the following way of solution for fault detection (Fig. 2):

1. Set-up a bipartite graph showing the structural properties of the system $\Sigma$.

2. Analyze the structure graph to find the structurally over-determined subsystem $\Sigma^{+}$.

3. Build a state observer for the subsystem $\Sigma^{+}$.

4. Use the state observer as residual generator to detect faults in the system $\Sigma$.

The main problem of these steps occur when transferring from Step 2 to Step 3, because it is not at all clear whether the model of the subsystem $\Sigma^{+}$can be used to design a residual generator. The main result of this paper shows under what condition observer-based diagnosis is possible for $\Sigma^{+}$.

For the derivation of the results, the system $\Sigma$ can be considered to be autonomous $(\boldsymbol{u}(t)=\mathbf{0})$, because known inputs do not influence structural properties of a linear system on the one hand and observability on the other hand. The autonomous system is abbreviated as $\Sigma=(\boldsymbol{A}, \boldsymbol{C})$.

\section{Theoretical background}

In this section, methods from structural diagnosability analysis as well as from structural control theory are explained. All necessary notions from the two branches of research, which will be important later, are introduced.

Notation. For a finite set $M$, the number of elements of $M$ is denoted by $|M|$. Let $n \in \mathbb{N}$. Then, $\underline{n}:=$ $\{1,2, \ldots, n\}$ is the set of natural numbers from 1 to $n$.

Given a matrix $\boldsymbol{A} \in \mathbb{R}^{n \times m}$ and index sets $I \subseteq$ $\{1, \ldots, n\}, J \subseteq\{1, \ldots, m\}$, the submatrix $\boldsymbol{A}(I, J)$ is received from $\boldsymbol{A}$ by deleting all rows $i \notin I$ and all columns $j \notin J$.

3.1. Structural analysis of fault detectability. This section applies the structural analysis methods summarised in Chapter 5 of the work of Blanke et al. (2016) to the linear system $\Sigma=(\boldsymbol{A}, \boldsymbol{C})$. The result is a method to find the structurally over-determined set of model equations, which will be denoted by $\mathcal{C}^{+}$. If such a set exists, faults are known to be structurally detectable.

3.1.1. Bipartite structure graph. For the structural analysis, the model $\Sigma=(\boldsymbol{A}, \boldsymbol{C})$ is interpreted as a set of equations

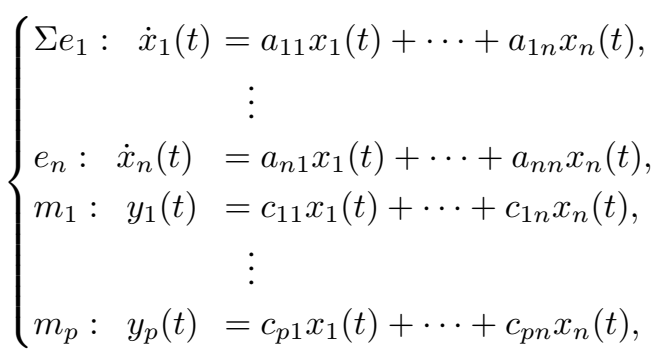

where $e_{i},(i=1, \ldots, n)$ or $m_{i},(i=1, \ldots, p)$ are the names of the state and output equations, respectively. If the system (2) with inputs is considered, then the right-hand sides of the equations $e_{i}$ have to be extended by the input signal terms $b_{i 1} u_{1}+\cdots+b_{i m} u_{m}(i=1, \ldots, n)$.

In structural analysis, variables are related to each other only via constraints. Thus, the relationship between $x_{i}(t)$ and its derivative $\dot{x}_{i}(t)$ has to be made explicit via the so-called differential constraints

$$
d_{i}: \quad \dot{x}_{i}(t)=\frac{\mathrm{d}}{\mathrm{d} t} x_{i}(t), \quad i=1,2, \ldots, n .
$$

In terms of structural analysis, the model equations $e_{i}, d_{i}$, and $m_{i}$ are said to be constraints that restrict the behavior of the system $\Sigma$. In this paper, the terms model equation and constraint and the terms variables and signals 
are used exchangeably. The set

$$
\mathcal{C}=\left\{d_{1}, d_{2}, \ldots, d_{n}, e_{1}, e_{2}, \ldots, e_{n}, m_{1}, m_{2}, \ldots, m_{p}\right\}
$$

represents the set of all constraints describing the system $\Sigma$. It is also called the model of $\Sigma$. The subset of differential constraints is denoted by $\mathcal{C}_{\mathrm{d}}:=\left\{d_{1}, \ldots, d_{n}\right\}$. The signals that appear in the constraints of the set $\mathcal{C}$ are the elements of the set

$$
\mathcal{Z}=\left\{\dot{x}_{1}, \dot{x}_{2}, \ldots, \dot{x}_{n}, x_{1}, x_{2}, \ldots, x_{n}, y_{1}, y_{2}, \ldots, y_{p}\right\}
$$

which is decomposed as $\mathcal{Z}=\mathcal{X} \cup \mathcal{K}$ with the set $\mathcal{X}=$ $\mathcal{X}_{*} \cup \dot{\mathcal{X}}$ of unknown internal signals

$$
\mathcal{X}_{*}:=\left\{x_{1}, \ldots, x_{n}\right\}, \quad \dot{\mathcal{X}}:=\left\{\dot{x}_{1}, \ldots, \dot{x}_{n}\right\},
$$

and the set of known signals $\mathcal{K}=\left\{y_{1}, \ldots, y_{p}\right\}$. If the model (2) or (3) is taken for the structural analysis, then, the constraint sets $\mathcal{Z}$ and $\mathcal{K}$ have to be extended by the known input variables $u_{1}, \ldots, u_{m}$ or the faults $f_{1}, \ldots, f_{l}$, respectively.

Step 1 mentioned in Section 2 will be investigated in the following. The property of being an over-determined system of equations is independent of any faults. Thus, to find over-determined equation sets, the model (4) of the fault-free system is used. Faults will become relevant in Step 2 which will be discussed later.

For a subset $C \subset \mathcal{C}$, the symbol $\operatorname{var}(C)$ defines the set of variables which appear in the equations of $C$, including known, unknown and fault variables. If a variable set $\mathcal{Y}$ is specified, then $\operatorname{var}_{\mathcal{Y}}(C):=\operatorname{var}(C) \cap \mathcal{Y}$.

In the bipartite structure graph $\mathcal{G}=(\mathcal{C}, \mathcal{X}, \mathcal{E})$ of the system $\Sigma$, the names $d_{i}, e_{i}$, and $m_{i}$ of the constraints are used as names of the vertices of the set $\mathcal{C}$, whereas the names of the signals are used for the elements of the vertex set $\mathcal{X}$. The set of edges $\mathcal{E} \subseteq \mathcal{C} \times \mathcal{X}$ is defined as follows:

$$
\left(c_{i}, z_{j}\right) \in \mathcal{E} \text { if } z_{j} \in \operatorname{var}\left(\left\{c_{i}\right\}\right)
$$

The left part of Fig. 3 shows an example, where the variable vertices $z_{j} \in \mathcal{Z}$ are shown as circles while the constraint vertices $c_{i} \in \mathcal{C}$ are drawn as bars.

The incidence matrix (biadjacency matrix) $\boldsymbol{G}$ of the graph $\mathcal{G}$ represents the graph as a set $\mathcal{E}$ of edges in an algebraic manner. The rows of this matrix are associated with the constraints and the columns with the variables. A " 1 " in the intersection of row $c_{i}$ and column $z_{j}$ indicates the existence of the edge $\left(c_{i}, z_{j}\right) \in \mathcal{E}$. All other elements are zero. The incidence matrix of $\Sigma$ has the structure given in Fig. 4. The dashed lines in the incidence matrix $\boldsymbol{G}$ separate the different classes of constraints or variables, respectively.

3.1.2. Analysis of the bipartite structure graph. According to Dulmage and Mendelsohn (1958) as well

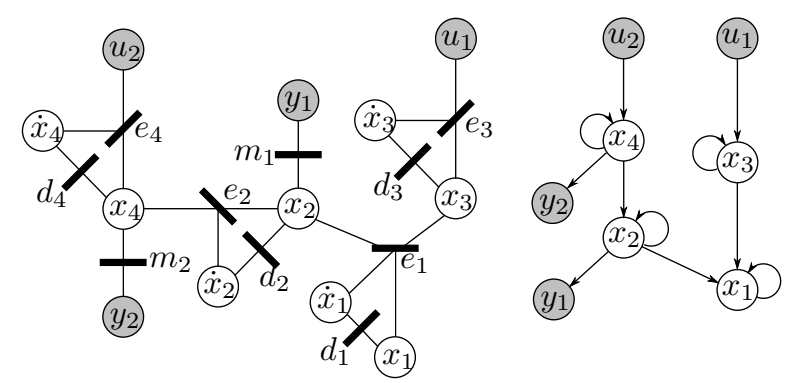

Fig. 3. Structure graphs of $\Sigma$.

as Pothen and Fan (1990), the incidence matrix of a bipartite graph can be brought into the block triangular form shown in Fig. 5 by re-ordering the rows and columns. This result is known as the Dulmage-Mendelsohn (DM) decomposition. In Fig. 5, the gray shaded areas include elements " 0 " and " 1 ", whereas in the white areas there are only zeros. The decomposition includes three unique disjoint subgraphs

$$
\begin{aligned}
& \mathcal{G}^{-}=\left(\mathcal{C}^{-}, \mathcal{X}^{-}, \mathcal{E}^{-}\right), \\
& \mathcal{G}^{0}=\left(\mathcal{C}^{0}, \mathcal{X}^{0}, \mathcal{E}^{0}\right), \\
& \mathcal{G}^{+}=\left(\mathcal{C}^{+}, \mathcal{X}^{+}, \mathcal{E}^{+}\right),
\end{aligned}
$$

with $\mathcal{X}=\mathcal{X}^{+} \cup \mathcal{X}^{0} \cup \mathcal{X}^{-}, \mathcal{C}=\mathcal{C}^{+} \cup \mathcal{C}^{0} \cup \mathcal{C}^{-}$. All vertex sets are pairwise disjoint and the following relations hold:

$$
\left|\mathcal{C}^{-}\right|<\left|\mathcal{X}^{-}\right|, \quad\left|\mathcal{C}^{0}\right|=\left|\mathcal{X}^{0}\right|, \quad\left|\mathcal{C}^{+}\right|>\left|\mathcal{X}^{+}\right|
$$

The naming of the equation sets is hence intuitive:

- $\mathcal{C}^{+}$is the structurally over-determined subset of equations,

- $\mathcal{C}^{0}$ is the structurally just-determined subset of equations,

- $\mathcal{C}^{-}$is the structurally under-determined subset of equations.

The structurally over-determined subset $\mathcal{C}^{+}$contains more equations than unknowns and, therefore, includes the redundancy which is crucial for fault diagnosis. The set $\mathcal{E}^{+}$ is the set of edges that appear between the nodes of $\mathcal{C}^{+}$ and the nodes of $\mathcal{X}^{+}$and is described by the dark gray rectangle in Fig.5 The submatrices on the block diagonal in Fig. 5] are the incidence matrices of the respective subgraphs $\mathcal{G}^{-}, \mathcal{G}^{0}$, and $\mathcal{G}^{+}$. As will be seen in Section 4 the subset $\mathcal{C}^{+}$of equations defines a state-space model $\Sigma^{+}$. This will be called the over-determined subsystem or the over-determined part of the system $\Sigma$.

The straight line in Fig. 5 shows "1"-elements that belong to a maximum matching of the graph and will be circled in the matrices as (1) (see Fig. 4). A matching $M \subset \mathcal{E}$ is a set of edges such that no two edges have 


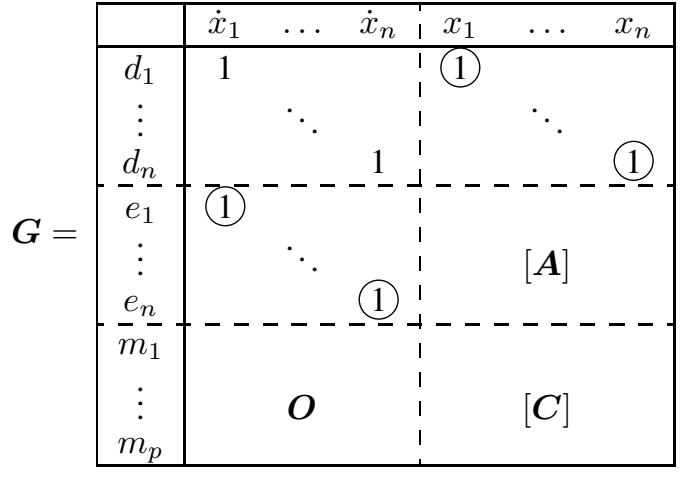

Fig. 4. Incidence matrix of $\mathcal{G}$.

a common vertex (Blanke et al., 2016). A matching is maximum if it is not possible to find another matching with a greater number of edges. A matching is $\mathcal{X}$-complete with respect to a vertex set $\mathcal{X}$ if all vertices of the set are covered by edges of the matching.

With respect to the incidence matrix of a bipartite graph, a matching is closely related to the structural rank of the matrix (Reinschke, 1988).

Definition 1. (Structural rank of a matrix) Given a matrix $\boldsymbol{G}$, a set of independent entries of $\boldsymbol{G}$ is defined as a set of nonzero entries no two of which lie on the same line (row or column). The structural rank ("s-rank") of $\boldsymbol{G}$ is defined as the maximum number of independent entries of $G$.

A matching of a graph $\mathcal{G}$ is closely related to the structural rank of $\boldsymbol{G}$ (see, e.g., the work of Murota (1987) for this).

Lemma 1. The cardinality of a maximum matching $M$ of a bipartite graph is equal to the structural rank of the corresponding incidence matrix, i.e.,

$$
|M|=\text { s-rank } \boldsymbol{G} .
$$

A way to find a maximum matching of a given bipartite graph is explained, e.g., by Blanke et al. (2016) or Pothen and Fan (1990). In the latter reference it is also explained how a maximum matching is used to compute the DM decomposition of the graph. It shall be mentioned that different maximum matchings yield the same DM decomposition.

For state-space models (2), it is always possible to choose a maximum matching of the following form:

$$
M=\left\{\left(d_{i}, x_{i}\right),\left(e_{i}, \dot{x}_{i}\right) \mid i=1, \ldots, n\right\} .
$$

The above matching shall be called the generic matching of the graph $\mathcal{G}$ of the system (2).

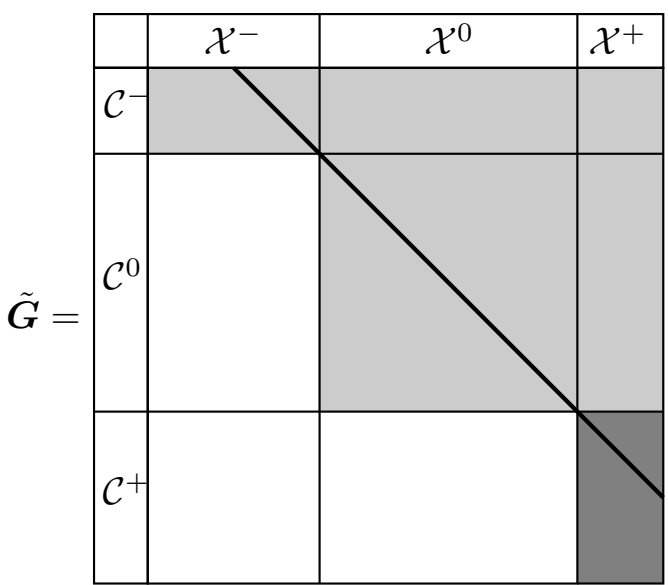

Fig. 5. Dulmage-Mendelsohn decomposition of the graph.

Given a matching $M$ in a graph, a path is called $M$-alternating if the edges in the path belong alternately to the matching and not to the matching. In terms of a maximum matching of $\mathcal{G}$, several vertex sets of a bipartite graph can be defined as follows (see Pothen and Fan, 1990):

$$
\begin{aligned}
\mathcal{X}^{+}:= & \{x \in \mathcal{X} \mid x \text { is reachable by an } M \text {-alternating } \\
& \text { path from some unmatched vertex } c \in \mathcal{C}\}, \\
\mathcal{C}^{+}:= & \{c \in \mathcal{C} \mid c \text { is reachable by an } M \text {-alternating } \\
& \text { path from some unmatched vertex } c \in \mathcal{C}\}, \\
\mathcal{X}^{-}:= & \{x \in \mathcal{X} \mid x \text { is reachable by an } M \text {-alternating } \\
& \text { path from some unmatched vertex } x \in \mathcal{X}\}, \\
\mathcal{C}^{-}:= & \{c \in \mathcal{C} \mid c \text { is reachable by an } M \text {-alternating } \\
& \text { path from some unmatched vertex } x \in \mathcal{X}\},
\end{aligned}
$$
and $\mathcal{C}^{0}:=\mathcal{C} \backslash\left(\mathcal{C}^{-} \cup \mathcal{C}^{+}\right), \mathcal{X}^{0}:=\mathcal{X} \backslash\left(\mathcal{X}^{-} \cup \mathcal{X}^{+}\right)$

Note that the generic matching (9) leaves no variable vertex $x \in \mathcal{X}$ unmatched, i.e., it is an $\mathcal{X}$-complete matching. Thus, the bipartite graph of any state-space model (2) only consists of the vertex sets $\mathcal{C}^{0}, \mathcal{C}^{+}, \mathcal{X}^{0}, \mathcal{X}^{+}$, and does not contain $\mathcal{C}^{-}, \mathcal{X}^{-}$. This is in accordance with the fact that any initial value problem (2) has a unique solution, and thus, cannot be under-determined.

3.1.3. Fault detectability. Fault detection is based on a test whether or not the measured signals are in consistency with the model $\mathcal{C}$ of the system $\Sigma$. This test is possible only if the system under consideration has more constraints than unknown variables. The structural analysis answers the question whether there exists an over-determined subset of equations. Accordingly, in the DM decomposition the over-determined set of equations $\mathcal{C}^{+}$represents the diagnosable part of the system. 
To evaluate the detectability of faults, a relation between the set of faults and the set of equations has to be used. It is generally assumed that any fault $f$ only changes a single constraint $c \in \mathcal{C}$. This assumption is used in the following definition (see, e.g., Blanke et al., 2016).

Definition 2. (Structural fault-detectability) A fault $f$ is said to be structurally fault-detectable in a model $\mathcal{C}$ if the following holds:

$$
\exists c \in \mathcal{C}^{+} \text {with } f \in \operatorname{var}(\{c\}) .
$$

The set of all structurally detectable faults is given by

$$
\mathcal{F}^{\mathrm{d}}:=\operatorname{var}_{\mathcal{F}}\left(\mathcal{C}^{+}\right) \subseteq \mathcal{F} .
$$

Note that structural detectability does not necessarily carry over to numerical detectability. That is, there may exist residual generators that provide vanishing residuals in the case of a fault. By nature, a structural property is necessary for the respective numerical property, but, in general, it is not sufficient. However, in this paper, it is assumed that a structurally detectable fault yields a residual deviating from zero and is, hence, detectable numerically by a diagnostic algorithm.

3.2. Structural analysis of observability. In this section, the notion of structural observability is introduced. It is closely related to the well-known $o b$ servability of dynamical systems. The advantage of the structural counterpart is that this property can be checked by means of a structure graph.

3.2.1. Directed structure graph. This section uses the notion of the system structure in the sense of the theory of structurally controllable and structurally observable systems. A class $\mathcal{S}(\boldsymbol{A}, \boldsymbol{C})$ of systems includes all systems $\Sigma=(\tilde{\boldsymbol{A}}, \tilde{\boldsymbol{C}})$ with matrices $\tilde{\boldsymbol{A}}$ and $\tilde{\boldsymbol{C}}$ that have the same structure as $\boldsymbol{A}$ or $\boldsymbol{C}$, respectively, according to the following definition.

Definition 3. (Structure of a matrix) Let $n, m \in \mathbb{N}$ and $\boldsymbol{M}=\left(\mu_{i j}\right) \in \mathbb{R}^{n \times m}$. Define the index set $J_{\boldsymbol{M}}:=$ $\left\{(i, j) \in\{1, \ldots, n\} \times\{1, \ldots, m\} \mid \mu_{i j}=0\right\}$ and $[\boldsymbol{M}]=\left(\hat{\mu}_{i j}\right) \in\{0,1\}^{n \times m}$ by $\hat{\mu}_{i j}=0$, if $(i, j) \in J_{\boldsymbol{M}}$, otherwise, $\hat{\mu}_{i j}=1$. $[\boldsymbol{M}]$ is called the structure of $\boldsymbol{M}$. Moreover, define

$$
\begin{aligned}
& \mathcal{S}(\boldsymbol{M}):=\left\{\boldsymbol{A}=\left(a_{i j}\right) \in \mathbb{R}^{n \times m} \mid a_{i j}=0\right. \\
&\text { if } \left.(i, j) \in J_{\boldsymbol{M}}\right\} .
\end{aligned}
$$

For two arbitrary matrices $M$ and $N$, we have

$$
\mathcal{S}(M, N):=\mathcal{S}(\boldsymbol{M}) \times \mathcal{S}(\boldsymbol{N}) .
$$

Structural observability of a system $\Sigma=(\boldsymbol{A}, \boldsymbol{C})$ is analyzed by a directed graph $\overrightarrow{\mathcal{G}}=(\overrightarrow{\mathcal{Z}}, \overrightarrow{\mathcal{E}})$ with the vertex set

$$
\overrightarrow{\mathcal{Z}}=\left\{x_{1}, x_{2}, \ldots, x_{n}, y_{1}, y_{2}, \ldots, y_{p}\right\}
$$

and the edge set $\overrightarrow{\mathcal{E}}$ that includes a directed edge $\overrightarrow{\left(x_{j}, x_{i}\right)}$ from vertex $x_{j}$ towards vertex $x_{i}$ if and only if in the constraint $e_{i}$ defined in (4) the coefficient $a_{i j}$ does not vanish $\left(\left[a_{i j}\right]=1\right)$. Furthermore, $\overrightarrow{\mathcal{E}}$ includes an edge $\overrightarrow{\left(x_{i}, y_{k}\right)}$ if and only if in the constraint $m_{k}$ the coefficient $c_{k i}$ does not vanish $\left(\left[c_{k i}\right]=1\right)$. The right part of Fig. 3 shows an example of a directed graph.

A state vertex $x_{i}$ is said to be output connected if there exists a directed path towards an output vertex $y_{j}$. Let $\overrightarrow{\mathcal{X}}=\left\{x_{1}, \ldots, x_{n}\right\}$. The set of all output connected state variables is denoted with $\overrightarrow{\mathcal{X}}_{\text {o }}$. The graph $\overrightarrow{\mathcal{G}}$ is output connected if all state vertices are output connected, i.e., $\overrightarrow{\mathcal{X}}=\overrightarrow{\mathcal{X}}_{\mathrm{o}}$. If $\overrightarrow{\mathcal{G}}$ corresponds to a linear system $\Sigma=(\boldsymbol{A}, \boldsymbol{C})$ and the former equality holds then $\Sigma$ is said to be output connected. For observability of a system, the output-connectedness of the corresponding directed graph is necessary, as will be seen in the following paragraph.

3.2.2. Structural observability. The notion of structural observability is defined as follows (Lunze, 2013; Reinschke, 1988).

Definition 4. (Structural observability) A system $\Sigma=$ $(\boldsymbol{A}, \boldsymbol{C})$ is called structurally observable if there is at least one system $(\tilde{\boldsymbol{A}}, \tilde{\boldsymbol{C}}) \in \mathcal{S}(\boldsymbol{A}, \boldsymbol{C})$ that is completely observable.

An important characteristic of structurally observable systems is the fact that "almost all" systems $\Sigma \in \mathcal{S}(\boldsymbol{A}, \boldsymbol{C})$ are completely observable. Hence, for almost all systems $\Sigma \in \mathcal{S}(\boldsymbol{A}, \boldsymbol{C})$ a state observer can be used for fault detection.

The above definition refers to the well-known notion of complete observability. A system $\Sigma$ is completely observable if and only if the observability matrix

$$
\mathcal{O}(\boldsymbol{A}, \boldsymbol{C})=\left(\begin{array}{c}
C \\
\boldsymbol{C A} \\
\vdots \\
C A^{n-1}
\end{array}\right)
$$

has full rank, i.e.,

$$
\operatorname{rank} \mathcal{O}(\boldsymbol{A}, \boldsymbol{C})=n
$$

If $\operatorname{rank} \mathcal{O}(\boldsymbol{A}, \boldsymbol{C})=s<n$, then there exists a transformation matrix $T \in \mathbb{R}^{n \times n}$ such that the matrices

$$
\tilde{A}:=T^{-1} A T, \quad \tilde{C}:=C T
$$

have block structure

$$
\tilde{\boldsymbol{A}}=\left(\begin{array}{ll}
\boldsymbol{A}_{1} & \boldsymbol{O} \\
\boldsymbol{A}_{2} & \boldsymbol{A}_{3}
\end{array}\right), \quad \tilde{\boldsymbol{C}}=\left(\begin{array}{ll}
\boldsymbol{C}_{1} & \boldsymbol{O}
\end{array}\right)
$$


with $\boldsymbol{A}_{1} \in \mathbb{R}^{s \times s}, \boldsymbol{C}_{1} \in \mathbb{R}^{p \times s}$ and the pair $\left(\boldsymbol{A}_{1}, \boldsymbol{C}_{1}\right)$ is completely observable. The new state vector is

$$
\tilde{\boldsymbol{x}}(t)=\left(\begin{array}{c}
\tilde{\boldsymbol{x}}_{1}(t) \\
\tilde{\boldsymbol{x}}_{2}(t)
\end{array}\right)=\boldsymbol{T}^{-1} \boldsymbol{x}(t)
$$

with $\tilde{\boldsymbol{x}}_{1}(t) \in \mathbb{R}^{s}$ and $\tilde{\boldsymbol{x}}_{2}(t) \in \mathbb{R}^{(n-s)}$. The vector $\tilde{\boldsymbol{x}}_{1}(t)$ represents the state vector of the completely observable subsystem.

Structural observability can be tested by the following necessary and sufficient condition (Lunze, 2013; Reinschke, 1988):

Theorem 1. A linear system $\Sigma=(\boldsymbol{A}, \boldsymbol{C})$ is structurally observable if and only if the following conditions are satisfied:

- $\quad \Sigma$ is output connected.

- $\operatorname{s-rank}\left(\begin{array}{l}\boldsymbol{A} \\ \boldsymbol{C}\end{array}\right)=n$.

\section{Relation between structural fault-detectability and structural observability}

This section provides the main result of the paper. At first, it is shown that both the output connected and the over-determined set of equations within a linear system define a state-space model. Moreover, the two subsystems coincide. Then, the results are used to formulate a criterion for checking structural observability in terms of the bipartite graph.

4.1. Output connected subsystem. In the literature (Lin, 1974; Reinschke, 1988; Shields and Pearson, 1976), the structural approach to observability (or controllability, respectively) was developed to provide a necessary condition for proving complete observability. No investigations have been made so far on what can be said about a system, which is not structurally observable. This gap will be closed in the following.

Consider the set of equations (4) representing $\Sigma$. If $\overrightarrow{\mathcal{X}}_{\mathrm{o}} \subset \overrightarrow{\mathcal{X}}$ is a proper subset of the state variable set, then define

$$
\mathcal{C}_{\mathrm{o}}:=\left\{m_{1}, \ldots, m_{p}\right\} \cup\left\{e_{i} \mid x_{i} \in \overrightarrow{\mathcal{X}}_{\mathrm{o}}\right\} .
$$

$\mathcal{C}_{\mathrm{o}}$ contains all output and all state equations which correspond to output connected variables.

Lemma 2. The set of equations $\mathcal{C}_{\mathrm{o}}$ defines a state-space model $\Sigma_{\mathrm{o}}$, which is called the output connected subsystem or the output connected part of $\Sigma$.

Proof. In a state-space model, each state variable $x_{i}(t)$ has to be declared by a first order differential equation. Thus, for each $x_{i}$ which appears in an equation of $\mathcal{C}_{\mathrm{o}}$, the respective equation $e_{i}$ has to be part of $\mathcal{C}_{0}$. Assume in the present situation that for an index $i, x_{i}$ belonged to $\operatorname{var}\left(\mathcal{C}_{\mathrm{o}}\right)$, but $e_{i} \notin \mathcal{C}_{\mathrm{o}}$. Then, by definition of $\mathcal{C}_{\mathrm{o}}, x_{i} \notin$ $\overrightarrow{\mathcal{X}}_{\mathrm{o}}$, and it appeared in some other equation $e_{j} \in \mathcal{C}_{\mathrm{o}}$ with $j \neq i$. From this followed that $\overrightarrow{\left(x_{i}, x_{j}\right)}$ were an edge in the directed graph related to $\mathcal{C}_{\mathrm{o}}$. But this would mean that $x_{i} \in \overrightarrow{\mathcal{X}}_{\mathrm{o}}$, which contradicts the assumption.

Let $q=\left|\overrightarrow{\mathcal{X}}_{\mathrm{o}}\right|$. The subsystem $\Sigma_{\mathrm{o}}$ is a pair $\left(\boldsymbol{A}_{\mathrm{o}}, \boldsymbol{C}_{\mathrm{o}}\right)$ of matrices with $\boldsymbol{A}_{\mathrm{o}} \in \mathbb{R}^{q \times q}, \boldsymbol{C}_{\mathrm{o}} \in \mathbb{R}^{p \times q}$. Define $I:=$ $\left\{i \in \underline{n} \mid x_{i} \in \overrightarrow{\mathcal{X}}_{\mathrm{o}}\right\}$. Then

$$
\boldsymbol{A}_{\mathrm{o}}=\boldsymbol{A}(I, I), \quad \boldsymbol{C}_{\mathrm{o}}=\boldsymbol{C}(\underline{p}, I) .
$$

Theorem 2. Given a linear system $\Sigma=(\boldsymbol{A}, \boldsymbol{C})$, let $\Sigma_{\mathrm{o}}=$ $\left(\boldsymbol{A}_{\mathrm{o}}, \boldsymbol{C}_{\mathrm{o}}\right)$ be the output connected subsystem of $\Sigma$ given by (20). The subsystem $\Sigma_{\mathrm{o}}$ is structurally observable if and only if

$$
\operatorname{s-rank}\left(\begin{array}{c}
\boldsymbol{A}_{\mathrm{o}} \\
\boldsymbol{C}_{\mathrm{o}}
\end{array}\right)=q
$$

Proof. If (21) holds, the two conditions of Theorem 1 are satisfied for $\Sigma_{0}$ and structural observability of $\Sigma_{0}$ follows immediately. The converse is obvious by Theorem 1

4.2. Over-determined subsystem. In the following it will be shown that the over-determined subset of equations $\mathcal{C}^{+}$within a state-space model defines a subsystem and that this subsystem equals the output connected subsystem. First, the relation between the vertex sets $\overrightarrow{\mathcal{X}}_{\mathrm{o}}$ and $\mathcal{X}^{+}$is studied.

Theorem 3. Let $\mathcal{X}_{*}^{+}:=\mathcal{X}^{+} \cap \mathcal{X}_{*}$. For a linear system (2) we have

$$
\mathcal{X}_{*}^{+}=\overrightarrow{\mathcal{X}}_{\mathrm{o}}
$$

The above theorem reveals the connection between the two graph-theoretic ways for analysing dynamic systems, namely bipartite graphs on the one hand and directed graphs on the other hand. It says that the state vertices which belong to the structurally over-determined subset of equations are exactly the output connected state vertices of the system.

Proof. In the following, the generic matching

$$
M=\left\{\left(d_{i}, x_{i}\right),\left(e_{i}, \dot{x}_{i}\right) \mid i=1, \ldots, n\right\}
$$

is used for argumentation (cf. Section 3.1.2).

(i) " $\mathcal{X}_{*}^{+} \subset \overrightarrow{\mathcal{X}}_{\mathrm{o}}$ ": Let $x \in \mathcal{X}_{*}^{+}$. By definition of the set $\mathcal{X}^{+}$ there exists an output equation $m_{l}$ and an $M$-alternating path in $\mathcal{G}$ that connects $m_{l}$ with $x$. In other words, there exists a sequence of state vertices $x_{i_{1}}, \ldots, x_{i_{k}}, x_{i_{k+1}}$ with 
$x_{i_{k+1}}=x$ such that the $M$-alternating path is of the form

$$
\begin{aligned}
m_{l}-x_{i_{1}} & \simeq d_{i_{1}}-\dot{x}_{i_{1}} \simeq e_{i_{1}}-x_{i_{2}} \\
& \simeq d_{i_{2}}-\dot{x}_{i_{2}} \simeq e_{i_{2}}-x_{i_{3}} \\
& \simeq \cdots-x_{i_{k}} \simeq d_{i_{k}}-\dot{x}_{i_{k}} \\
& \simeq e_{i_{k}}-x_{i_{k+1}}
\end{aligned}
$$

where the symbol $\simeq$ denotes the matched edges. Hence, for all $j=1, \ldots, k$, the inclusion $x_{i_{j+1}} \in \operatorname{var}\left(\left\{e_{i_{j}}\right\}\right)$ holds. Equivalently, the entry $a_{i_{j} i_{j+1}}$ in the matrix $\boldsymbol{A}$ is nonzero for all $j=1, \ldots, k$, and thus, there exists a directed edge $\overrightarrow{\left(x_{i_{j+1}}, x_{i_{j}}\right)}$ in the graph $\overrightarrow{\mathcal{G}}$. Moreover, since $x_{i_{1}} \in \operatorname{var}\left(\left\{m_{l}\right\}\right)$, the entry $c_{l i_{1}}$ in the matrix $\boldsymbol{C}$ is nonzero, and so, there exists a directed edge $\overrightarrow{\left(x_{i_{1}}, y_{l}\right)}$ in $\overrightarrow{\mathcal{G}}$. Therefore, there exists a directed path

$$
x_{i_{k+1}} \rightarrow x_{i_{k}} \rightarrow x_{i_{k-1}} \rightarrow \cdots \rightarrow x_{i_{2}} \rightarrow x_{i_{1}} \rightarrow y_{l}
$$

in the directed graph $\overrightarrow{\mathcal{G}}$ which connects $x$ and $y_{l}$. Thus, $x \in \overrightarrow{\mathcal{X}}_{\mathrm{o}}$.

(ii) " $\overrightarrow{\mathcal{X}}_{\mathrm{o}} \subset \mathcal{X}_{*}^{+}$": Suppose $x \in \vec{X}_{\mathrm{o}}$ and there exists a directed path in the directed graph $\overrightarrow{\mathcal{G}}$ which connects $x$ with an output vertex $y_{l}$, as indicated in (23). Then, by the same arguments as before, there exists an $M$-alternating path (22) in $\mathcal{G}_{\text {red }}$ that connects $m_{l}$ and $x$. Thus, $x \in \mathcal{X}_{*}^{+}$, which proves the assertion.

Corollary 1. We have that

$$
\mathcal{C}^{+}=\left\{m_{1}, \ldots, m_{p}\right\} \cup\left\{e_{i}, d_{i} \mid x_{i} \in \mathcal{X}_{*}^{+}\right\} .
$$

Let $n^{+}=\left|\mathcal{X}_{*}^{+}\right|$. The equations of $\mathcal{C}^{+}$define a state-space model

$$
\Sigma^{+}=\left(\boldsymbol{A}^{+}, \boldsymbol{C}^{+}\right)
$$

with $\boldsymbol{A}^{+} \in \mathbb{R}^{n^{+} \times n^{+}}, \boldsymbol{C}^{+} \in \mathbb{R}^{p \times n^{+}}$, which will be called the over-determined subsystem of $\Sigma$.

Proof. Given the generic matching $M$ as in the proof above, it is clear that a state vertex $x_{i}$ belongs to $\mathcal{X}^{+}$if and only if the corresponding equation vertex $e_{i}$ belongs to $\mathcal{C}^{+}$. This holds since an $M$-alternating path ending in $x_{i}$ can always be enlarged by the sequence $x_{i} \simeq d_{i}-$ $\dot{x}_{i} \simeq e_{i}$, such that the vertex $e_{i}$ is also covered by the alternating path and, therefore, belongs to $\mathcal{C}^{+}$. Moreover, all output equations $m_{1}, \ldots, m_{p}$ belong to $\mathcal{C}^{+}$since the generic matching $M$ leaves these equations unmatched.

The subsystem matrices $\boldsymbol{A}^{+}$and $\boldsymbol{C}^{+}$can be derived similarly to those of the output connected subsystem. Let $I:=\left\{i \in \underline{n} \mid x_{i} \in \mathcal{X}_{*}^{+}\right\}$. Then for $\boldsymbol{A}^{+}$and $\boldsymbol{C}^{+}$the following equalities hold:

$$
\boldsymbol{A}^{+}=\boldsymbol{A}(I, I), \quad \boldsymbol{C}^{+}=\boldsymbol{C}(\underline{p}, I) .
$$

For the subsystem $\Sigma_{\mathrm{o}}$ given by (20) and the equalities (26), the following corollary is immediate.
Corollary 2. For a linear system (2)

$$
\Sigma^{+}=\Sigma_{\mathrm{o}}
$$

i.e., the over-determined subsystem given by (26) equals the output-connected subsystem given by (20).

4.3. Structural observability of $\boldsymbol{\Sigma}^{+}$. In the following we shall investigate under what condition the over-determined subsystem $\Sigma^{+}$is structurally observable. A criterion for structural observability is provided in terms of the bipartite graph of the system $\Sigma$.

Before the subsequent corollary clarifies the relation between observability and diagnosability of a system, remind that the structurally over-determined part of a system is called diagnosable.

Corollary 3. Given a linear system $\Sigma=(\boldsymbol{A}, \boldsymbol{B}, \boldsymbol{C})$, the following implications hold:

$$
\begin{aligned}
& \sum \text { is completely observable } \\
& \quad \Rightarrow \Sigma \text { is structurally observable } \\
& \quad \Rightarrow \Sigma \text { is structurally over-determined. }
\end{aligned}
$$

Proof. The first implication holds due to Definition 4 The second implication holds due to Theorems 1 and 3 . If the system is structurally observable, then it is in particular output connected, and, therefore, structurally over-determined.

Lemma 3. Let $\Sigma=(\boldsymbol{A}, \boldsymbol{C})$ be given and $\Sigma^{+}=$ $\left(\boldsymbol{A}^{+}, \boldsymbol{C}^{+}\right)$be the over-determined subsystem of $\Sigma . \Sigma^{+}$ is structurally observable if and only if

$$
\text { s-rank }\left(\begin{array}{l}
\boldsymbol{A}^{+} \\
\boldsymbol{C}^{+}
\end{array}\right)=n^{+} \text {. }
$$

Proof. The claim follows immediately from Theorems 2 and 2 .

Condition (28) can also be expressed in terms of bipartite graphs by using the notion of a causal matching:

Definition 5. (Causal matching) Given the bipartite structure graph $\mathcal{G}$ of the system (2), a matching of $\mathcal{G}$ is said to be causal if it does not contain any of the edges $\left(d_{i}, x_{i}\right), i=1, \ldots, n$.

The above definition refers to the causal interpretation of differential constraints in a bipartite graph and has been studied by, e.g., Blanke et al. (2016), Bregon et al. (2014) or Frisk et al. (2012). Note that a causal matching in the present context is equivalent to derivative causality in the mentioned references. Now, observe the following relationship.

Lemma 4. For a given linear system $\Sigma=(\boldsymbol{A}, \boldsymbol{C})$ let $\mathcal{G}=(\mathcal{C}, \mathcal{X}, \mathcal{E})$ be the corresponding bipartite graph. Then the following two conditions are equivalent: 
(i) There exists an $\mathcal{X}$-complete causal matching of $\mathcal{G}$.

(ii) $\operatorname{s-rank}\left(\begin{array}{l}\boldsymbol{A} \\ \boldsymbol{C}\end{array}\right)=n$.

Proof. The above result becomes clear if Fig. 4 is taken into account. A formal proof is provided subsequently.

Suppose that $\mathcal{G}$ possesses an $\mathcal{X}$-complete causal matching $M \subseteq \mathcal{E}$. In particular, such a matching is maximum. In $(8)$ it was seen that s-rank $G$ is equal to the cardinality of a maximum matching: s-rank $\boldsymbol{G}=|M|$. Since $M$ is causal and $\mathcal{X}$-complete, the vertices in $\mathcal{X}_{*}:=$ $\left\{x_{1}, \ldots, x_{n}\right\}$ have to be matched by constraints of the set $\mathcal{C}_{*}:=\left\{e_{1}, \ldots, e_{n}\right\} \cup\left\{m_{1}, \ldots, m_{p}\right\}$. This is equivalent to

$$
\operatorname{s-rank}\left(\begin{array}{l}
\boldsymbol{A} \\
\boldsymbol{C}
\end{array}\right)=n \text {. }
$$

For the converse, assume that (ii) is fulfilled. Then, the vertices in $\mathcal{X}_{*}$ can be matched by vertices in $\mathcal{C}_{*}$. Such a matching can be augmented by the edges $\left(d_{i}, \dot{x}_{i}\right)$, $i=1, \ldots, n$. The resulting matching is $\mathcal{X}$-complete and causal which completes the proof.

A combination of the preceding results yields the following main result of this paper.

Theorem 4. Let $\Sigma=(\boldsymbol{A}, \boldsymbol{C})$ be given and $\mathcal{G}=$ $(\mathcal{C}, \mathcal{X}, \mathcal{E})$ be the corresponding bipartite graph. The overdetermined subsystem $\Sigma^{+}=\left(\boldsymbol{A}^{+}, \boldsymbol{C}^{+}\right)$is structurally observable if and only if $\mathcal{G}^{+}=\left(\mathcal{C}^{+}, \mathcal{X}^{+}, \mathcal{E}^{+}\right)$possesses an $\mathcal{X}^{+}$-complete causal matching.

Moreover, if $\mathcal{G}$ possesses an $\mathcal{X}$-complete causal matching, then $\mathcal{G}^{+}$possesses an $\mathcal{X}^{+}$-complete causal matching.

Proof. The first part follows from Lemma 4, Corollary 2 and Theorem $1 . \quad$ For the second part, let $M$ be an $\mathcal{X}$-complete causal matching of $\mathcal{G}$. Such a matching is maximum and, hence, a DM decomposition can be computed from $M$ which yields $\mathcal{C}=\mathcal{C}^{0} \cup \mathcal{C}^{+}$and $\mathcal{X}=\mathcal{X}^{0} \cup \mathcal{X}^{+}$. Since $M$ is causal, each of the vertices $x_{i} \in \mathcal{X}_{*}$ has to be matched by a constraint of the set $\mathcal{C} \backslash \mathcal{C}_{\mathrm{d}}=\left\{e_{1}, \ldots, e_{n}\right\} \cup\left\{m_{1}, \ldots, m_{p}\right\}$. By the properties of a DM decomposition, the variables in $\mathcal{X}^{+}$are matched by the equations in $\mathcal{C}^{+}$. In all, the variables in $\mathcal{X}_{*}^{+}$are matched by equations in $\mathcal{C}^{+} \backslash \mathcal{C}_{\mathrm{d}}$. This proves the assertion.

This result has the important consequence that fault detection systems can be obtained by first analyzing the bipartite structure graph of a system to find the over-determined part $\mathcal{C}^{+}$of the model $\mathcal{C}$ and, second, by using the model $\mathcal{C}^{+}$to create a state observer that generates non-vanishing residuals if a fault associated with the constraints in the set $\mathcal{C}^{+}$occurs (cf. Section 5).

The present section will be concluded with a study of the case where the system $\Sigma$ is given in its observability decomposition.
Lemma 5. For a linear system $\tilde{\Sigma}$ in its observability decomposition (16),

$$
\overrightarrow{\mathcal{X}}_{\mathrm{o}}=\left\{\tilde{x}_{1}, \ldots, \tilde{x}_{s}\right\}
$$

that is, the output connected variables of $\tilde{\Sigma}$ are exactly the components of $\tilde{\boldsymbol{x}}_{1}$.

Proof. Since the subsystem $\Sigma_{\text {obs }}$ is completely observable, it is in particular structurally observable. Hence, the state variables of this subsystem are output connected. Therefore, $\left\{\tilde{x}_{1}, \ldots, \tilde{x}_{s}\right\} \subseteq \overrightarrow{\mathcal{X}}_{\text {o. }}$. On the other hand, due to the structure of the matrices $\tilde{A}$ and $\tilde{\boldsymbol{C}}$, no state variable of $\tilde{\boldsymbol{x}}_{2}$ can be output connected. Thus, we get (29).

Corollary 3 says that the property of a linear system of being structurally over-determined is weaker than the property of being structurally observable or completely observable, respectively. The next result asserts that the three properties are equivalent if the system is given in its observability decomposition.

Theorem 5. Consider a linear system $\Sigma=(\boldsymbol{A}, \boldsymbol{C})$ that is given in its observability decomposition (16) with

$$
\boldsymbol{A}=\left(\begin{array}{ll}
\boldsymbol{A}_{1} & \boldsymbol{O} \\
\boldsymbol{A}_{2} & \boldsymbol{A}_{3}
\end{array}\right), \quad \boldsymbol{C}=\left(\begin{array}{ll}
\boldsymbol{C}_{1} & \boldsymbol{O}
\end{array}\right)
$$

and $\Sigma_{\mathrm{obs}}=\left(\boldsymbol{A}_{1}, \boldsymbol{C}_{1}\right)$ being a completely observable subsystem. Moreover, let $\Sigma^{+}$be an over-determined subsystem. Then

$$
\Sigma_{\mathrm{obs}}=\Sigma^{+}
$$

Proof. By Lemma 5, the output connected state variables are precisely those of the subsystem $\Sigma_{\text {obs }}$. That is, $\Sigma_{\text {obs }}=$ $\Sigma_{\mathrm{o}}$. The claim follows from Corollary 2

\section{Comprehensive method to design an observer-based fault detector}

The results of the preceding section show how fault-detectability is related to observability of a linear system and, thus, indicate how an observer-based fault detection system can be built. The set $\mathcal{C}^{+}$of model equations forms the state-space model

$$
\Sigma^{+}:\left\{\begin{aligned}
\dot{\boldsymbol{x}}^{+}(t) & =\boldsymbol{A}^{+} \boldsymbol{x}^{+}(t)+\boldsymbol{B}^{+} \boldsymbol{u}(t), \\
\boldsymbol{x}^{+}(0) & =\boldsymbol{x}_{0}^{+} \\
\boldsymbol{y}(t) & =\boldsymbol{C}^{+} \boldsymbol{x}^{+}(t) .
\end{aligned}\right.
$$

with $\boldsymbol{A}^{+}, \boldsymbol{C}^{+}$as in $(26)$ and $\boldsymbol{B}^{+}=\boldsymbol{B}(I, \underline{m})$. Theorem 4 says that if there exists an $\mathcal{X}^{+}$-complete causal matching of the bipartite graph $\mathcal{G}^{+}$of the above system, then $\Sigma^{+}$is structurally observable. That is, for almost all parameters occurring in the matrices $\boldsymbol{A}^{+}$and $\boldsymbol{C}^{+}$the pair $\left(\boldsymbol{A}^{+}, \boldsymbol{C}^{+}\right)$ 
is completely observable according to the condition (14) and a state observer can be used for fault detection. The Luenberger observer that belongs to the system $\Sigma^{+}$has the form

$$
O:\left\{\begin{array}{l}
\dot{\hat{\boldsymbol{x}}}(t)=\left(\boldsymbol{A}^{+}-\boldsymbol{L} \boldsymbol{C}^{+}\right) \hat{\boldsymbol{x}}(t)+\boldsymbol{B}^{+} \boldsymbol{u}(t)+\boldsymbol{L} \boldsymbol{y}(t), \\
\hat{\boldsymbol{x}}(0)=\hat{\boldsymbol{x}}_{0}, \\
\boldsymbol{r}(t)=\boldsymbol{y}(t)-\boldsymbol{C}^{+} \hat{\boldsymbol{x}}(t) .
\end{array}\right.
$$

It delivers the residual $\boldsymbol{r}(t)$, which has to be evaluated for fault detection (Fig. 1). The feedback matrix $\boldsymbol{L}$ has to be selected so as to make the system given in (32) asymptotically stable, i.e., to shift all eigenvalues of $\boldsymbol{A}^{+}-\boldsymbol{L} \boldsymbol{C}^{+}$to the complex left half-plane. Such a feedback matrix exists if the pair $\left(\boldsymbol{A}^{+}, \boldsymbol{C}^{+}\right)$is completely observable (Lunze, 2013).

If now a fault occurs which changes the dynamic behavior of the subsystem $\Sigma^{+}$, then the estimation error $\boldsymbol{r}(t)$ of the observer will not converge to zero. Hence, the state observer is sensitive to faults $f \in \mathcal{F}^{\mathrm{d}}$ (cf. (11)). For residual evaluation, a norm $\|\cdot\|$ on $\mathbb{R}^{p}$ and a threshold $\varepsilon>0$ have to be determined. Once this is done, the residual vector is evaluated as follows:

- If $\|\boldsymbol{r}(t)\| \leq \varepsilon$, then the system $\Sigma$ is supposed to work normally.

- If $\|\boldsymbol{r}(t)\|>\varepsilon$ for some $t>0$, then the system is faulty.

The threshold $\varepsilon$ is necessary since the residual will in practice always deviate from zero due to disturbances or model uncertainties.

In summary, the way to design observer-based fault detection systems, which has been outlined in Section 2 , can now be presented in more detail as Algorithm 1.

\begin{tabular}{l}
\hline Algorithm 1. Design of a residual generator. \\
Given: Model (2) \\
Step 1. Set-up the bipartite structure graph $\mathcal{G}=(\mathcal{C}, \mathcal{X}, \mathcal{E})$ \\
and the corresponding incidence matrix $G($ see Fig. 4 )
\end{tabular}

Step 2. Apply the DM decomposition to $\boldsymbol{G}$ to find the structurally over-determined model $\mathcal{C}^{+}$.

Step 3. Test whether there exists an $\mathcal{X}^{+}$-complete causal matching of $\mathcal{G}^{+}$.

Step 4. If the test is positive, set up the subsystem $\Sigma^{+}$(see (25)) and build a state observer (32).

Result: Residual generator.

Together with a residual evaluation system, this residual generator represents a fault detection system for $\Sigma$ that detects fault in the detectable fault set $\mathcal{F}_{\mathrm{d}}$.

The main result of this paper shows how structural diagnosability analysis can be combined with observer-based diagnosis. Steps 1-3 of Algorithm 1

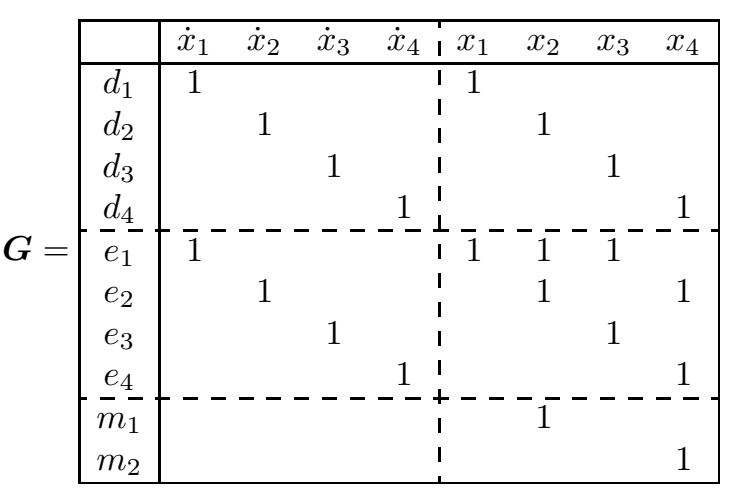

Fig. 6. Incidence matrix of $\Sigma$.

use the bipartite structure graph to find the diagnosable part. Under the condition stated in Theorem 4 an observer-based diagnostic unit can be designed for this part. The analysis of the bipartite graph simultaneously shows the structural observability, which has been expressed in the literature by an alternative structural representation in terms of a directed graph. Hence, no separate analysis with respect to observability is necessary.

\section{Example}

Consider the system (2) with

$$
\begin{gathered}
\boldsymbol{A}=\left(\begin{array}{cccc}
a_{11} & a_{12} & a_{13} & 0 \\
0 & a_{22} & 0 & a_{24} \\
0 & 0 & a_{33} & 0 \\
0 & 0 & 0 & a_{44}
\end{array}\right), \quad \boldsymbol{B}=\left(\begin{array}{cc}
0 & 0 \\
0 & 0 \\
b_{31} & 0 \\
0 & b_{42}
\end{array}\right), \\
\boldsymbol{C}=\left(\begin{array}{cccc}
0 & c_{12} & 0 & 0 \\
0 & 0 & 0 & c_{24}
\end{array}\right),
\end{gathered}
$$

where all parameters are assumed to be nonzero. Further, the following faults may occur:

- $f_{1}(t)$ : fault of the actuator belonging to the input $u_{1}$,

- $f_{2}(t)$ : fault of the actuator belonging to the input $u_{2}$,

- $f_{3}(t)$ : fault of the sensor belonging to the output $y_{1}$,

- $f_{4}(t)$ : fault of the sensor belonging to the output $y_{2}$. These faults lead to the matrices

$$
\boldsymbol{E}_{\mathrm{s}}=\left(\begin{array}{cccc}
0 & 0 & 0 & 0 \\
0 & 0 & 0 & 0 \\
1 & 0 & 0 & 0 \\
0 & 1 & 0 & 0
\end{array}\right), \quad \boldsymbol{E}_{\mathrm{o}}=\left(\begin{array}{cccc}
0 & 0 & 1 & 0 \\
0 & 0 & 0 & 1
\end{array}\right)
$$

For the system $\Sigma$, we have $\operatorname{rank} \mathcal{O}(A, C) \leq 2<4$. Thus, it is not completely observable. Nevertheless, the application of Algorithm 1 will show that there is a diagnosable part for which observer-based diagnostic methods can be applied. 
Step 1: The structure graphs $\mathcal{G}$ and $\overrightarrow{\mathcal{G}}$ are shown in Fig. 3 and the corresponding incidence matrix $\boldsymbol{G}$ in Fig.6, The known input and output variables are included in the graphs and indicated by grey circles.

Step 2: The DM decomposition of the graph $\mathcal{G}$ results in the following incidence matrix $\hat{G}$ :

$\hat{\boldsymbol{G}}=$\begin{tabular}{|c|cccc|cccc|}
\hline & $\dot{x}_{1}$ & $x_{1}$ & $\dot{x}_{3}$ & $x_{3}$ & $\dot{x}_{2}$ & $\dot{x}_{4}$ & $x_{2}$ & $x_{4}$ \\
\hline$d_{1}$ & 1 & 1 & 0 & 0 & 0 & 0 & 0 & 0 \\
$e_{1}$ & 1 & 1 & 0 & 1 & 0 & 0 & 1 & 0 \\
$d_{3}$ & 0 & 0 & 1 & 1 & 0 & 0 & 0 & 0 \\
$e_{3}$ & 0 & 0 & 1 & 1 & 0 & 0 & 0 & 0 \\
\hline$d_{2}$ & & & & & 1 & 0 & 1 & 0 \\
$d_{4}$ & & & & & 0 & 1 & 0 & 1 \\
$e_{2}$ & & & & & 1 & 0 & 1 & 1 \\
$e_{4}$ & & & & & 0 & 1 & 0 & 1 \\
$m_{1}$ & & & & & 0 & 0 & 1 & 0 \\
$m_{2}$ & & & & & 0 & 0 & 0 & 1 \\
\hline
\end{tabular}

The over-determined set of equations is hence given by $\mathcal{C}^{+}=\left\{e_{2}, e_{4}, d_{2}, d_{4}, m_{1}, m_{2}\right\}$.

Step 3: The incidence matrix $\boldsymbol{G}^{+}$of the over-determined subgraph $\mathcal{G}^{+}$is the submatrix down right in the matrix $\hat{\boldsymbol{G}}$ above. $M=\left\{\left(d_{i}, \dot{x}_{i}\right),\left(e_{i}, x_{i}\right) \mid i=2,4\right\}$ is an $\mathcal{X}^{+}$-complete causal matching of the bipartite graph $\mathcal{G}^{+}$. This matching is indicated by the circled entries in the above matrix. Thus, the over-determined subsystem $\Sigma^{+}$ is structurally observable by Theorem 4

Step 4: The related subsystem $\Sigma^{+}$is defined by

$$
\Sigma^{+}:\left\{\begin{aligned}
\dot{x}_{2}(t) & =a_{22} x_{2}(t)+a_{24} x_{4}(t) \\
\dot{x}_{4}(t) & =a_{44} x_{4}(t)+b_{42} u_{2}(t) \\
y_{1}(t) & =c_{12} x_{2}(t) \\
y_{2}(t) & =c_{24} x_{4}(t) .
\end{aligned}\right.
$$

The subsystem matrices $\boldsymbol{A}^{+}, \boldsymbol{B}^{+}$, and $\boldsymbol{C}^{+}$are

$$
\begin{gathered}
\boldsymbol{A}^{+}=\left(\begin{array}{cc}
a_{22} & a_{24} \\
0 & a_{44}
\end{array}\right), \quad \boldsymbol{B}^{+}=\left(\begin{array}{cc}
0 & 0 \\
0 & b_{42}
\end{array}\right), \\
\boldsymbol{C}^{+}=\left(\begin{array}{cc}
c_{12} & 0 \\
0 & c_{24}
\end{array}\right) .
\end{gathered}
$$

$\Sigma^{+}$is also completely observable according to (14) since $c_{12}$ and $c_{24}$ are assumed to be nonzero. Thus, an observer can be built for $\Sigma^{+}$. Such an observer is able to detect faults that affect the over-determined subsystem. Recalling Definition 2, these are subsumed in the set $\mathcal{F}^{\mathrm{d}}=\left\{f_{2}, f_{3}, f_{4}\right\}$. By contrast, $f_{1}$ which affects equation $e_{3}$ is not detectable since $e_{3}$ does not belong to the over-determined part and is not used by the observer.
Consider a numerical realization of the system $\Sigma$ with the following parameters:

$$
\begin{array}{lll}
a_{11}=-0.1, & a_{12}=0.6, & a_{13}=-0.3, \\
a_{22}=-2.2, & a_{24}=1.5, & a_{33}=-0.5, \\
a_{44}=-0.2, & b_{31}=1.4, & b_{42}=0.5, \\
c_{12}=1, & c_{24}=1 . &
\end{array}
$$

A Luenberger observer, as given in (32), can be established for the given system with, e.g.,

$$
\boldsymbol{L}=\left(\begin{array}{cc}
0.1 & 1.5 \\
0 & 0.1
\end{array}\right)
$$

where the above matrix has been found by a pole placement procedure (see Lunze, 2013). The initial state $\hat{\boldsymbol{x}}_{0}$ of the observer has been set to $(0,0)^{\mathrm{T}}$.
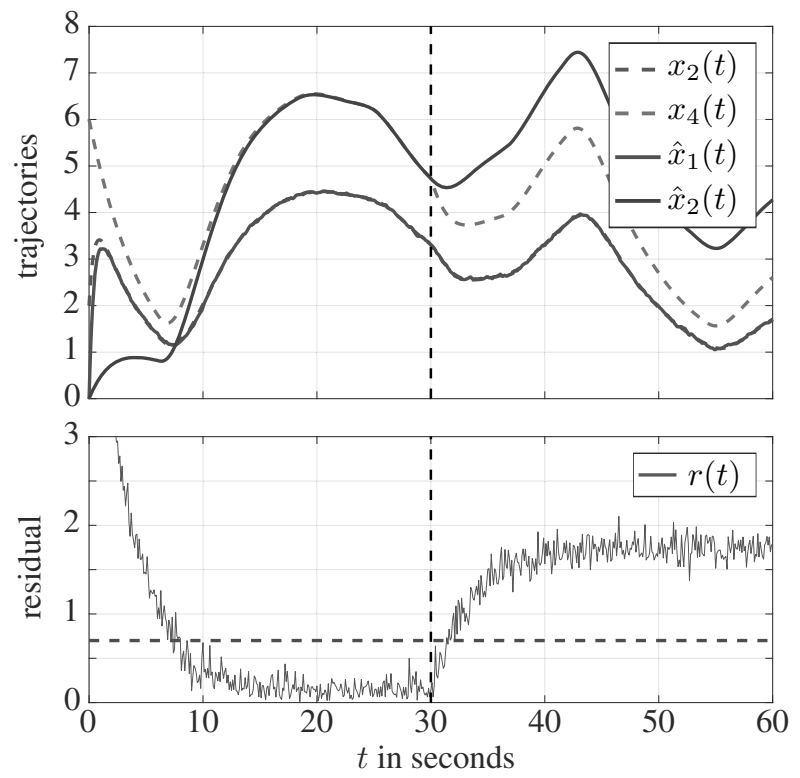

Fig. 7. State trajectories and residual evolution.

The upper part of Fig. 7 shows the trajectories of $x_{2}(t)$ and $x_{4}(t)$ as dashed lines which are the state of the over-determined subsystem $\Sigma^{+}$. The solid lines correspond to the state $\hat{\boldsymbol{x}}(t)=\left(\hat{x}_{1}(t), \hat{x}_{2}(t)\right)^{\mathrm{T}}$ of the observer. The observer state $\hat{\boldsymbol{x}}(t)$ converges to $\boldsymbol{x}^{+}(t)=$ $\left(x_{2}(t), x_{4}(t)\right)$ in the fault-free case. Likewise, the residual converges to zero if no fault occurs. This is depicted in the lower part of Fig. 7 It shows the evolution of the norm $\|\boldsymbol{r}(t)\|$ of the residual. The latter is given by

$$
\boldsymbol{r}(t)=\boldsymbol{y}(t)-\boldsymbol{C} \hat{\boldsymbol{x}}(t) .
$$

The 2-norm $\|\boldsymbol{r}(t)\|=\sqrt{r_{1}(t)^{2}+r_{2}(t)^{2}}$ was chosen and the threshold for residual evaluation has been set to $\varepsilon=$ 0.7 . 
At $t=30 \mathrm{~s}$ a fault has been injected. This is indicated by the vertical dashed lines in both plots. The observer-based residual reacts to this fault and exceeds the threshold at $t \approx 32 \mathrm{~s}$. Thus, the fault is being detected successfully.

\section{Conclusion}

The paper has shown how structural analysis of fault-detectability can be combined with designing fault detection systems by means of observers that operate under practical circumstances like disturbances. The main result is the relation between the structurally over-determined part of a linear system that can be obtained by the bipartite structure graph and the structural observability of the system that can be investigated by a directed structure graph (Theorem 4).

An extension from fault detection to fault isolation needs the use the notion of proper structurally over-determined (PSO) sets (Krysander et al., 2008). For these sets, conditions have to be derived that assure that a PSO set defines a structurally observable subsystem. For a number of observable PSO subsystems, a bank of observers could be built that allowed structural fault isolation via a fault signature matrix.

\section{References}

Armengol, J., Bregón, A., Escobet, T., Gelso, E., Krysander, M., Nyberg, M., Olive, X., Pulido, B. and Travé-Massuyès, L. (2009). Minimal structurally overdetermined sets for residual generation: A comparison of alternative approaches, 7th IFAC Symposium on Fault Detection, Supervision and Safety of Technical Processes, Barcelona, Spain, pp. 1480-1485.

Blanke, M., Kinnaert, M., Lunze, J. and Staroswiecki, M. (2016). Diagnosis and Fault-Tolerant Control, 3rd Edn., Springer, Berlin.

Boukhobza, T., Hamelin, F. and Sauter, D. (2006). Observability of structured linear systems in descriptor form: A graph-theoretic approach, Automatica 42(4): 629-635.

Boukhobza, T., Hamelin, F. and Martinez-Martinez, S. (2007). State and input observability for structured linear systems: A graph-theoretic approach, Automatica 43(7): 1204-1210.

Bregon, A., Alonso-Gonzalez, C.J. and Pulido, B. (2014). Integration of simulation and state observers for online fault detection of nonlinear continuous systems, IEEE Transactions on Systems, Man, and Cybernetics: Systems 44(12): 1553-1568.

Commault, C. and Dion, J.M. (2007). Sensor location for diagnosis in linear systems: A structural analysis, IEEE Transactions on Automatic Control 52(2): 155-169.

De Persis, C. and Isidori, A. (2001). A geometric approach to nonlinear fault detection and isolation, IEEE Transactions on Automatic Control 46(6): 853-865.
Ding, S.X. (2013). Model-Based Fault Diagnosis Techniques, 2nd Edn., Springer, Berlin.

Dulmage, A.L. and Mendelsohn, N.S. (1958). Coverings of bipartite graphs, Canadian Journal of Mathematics 10: 517-534.

Frisk, E., Bregon, A., Aslund, J., Krysander, M., Pulido, B. and Biswas, G. (2012). Diagnosability analysis considering causal interpretations for differential constraints, IEEE Transactions on Systems, Man and Cybernetics A: Systems and Humans 42(5): 1216-1229.

Isermann, R. (2006). Fault-Diagnosis Systems: An Introduction from Fault Detection to Fault Tolerance, Springer, Berlin.

Krysander, M. (2006). Design and Analysis of Diagnosis Systems Using Structural Methods, PhD thesis, Linköpings University, Linköpings.

Krysander, M. and Frisk, E. (2008). Sensor placement for fault diagnosis, IEEE Transactions on Systems, Man and Cybernetics A: Systems and Humans 38(6): 1398-1410.

Krysander, M., Aslund, J. and Nyberg, M. (2008). An efficient algorithm for finding minimal overconstrained subsystems for model-based diagnosis, IEEE Transactions on Systems, Man and Cybernetics A: Systems and Humans 38(1): 197-206.

Lin, C.-T. (1974). Structural controllability, IEEE Transactions on Automatic Control 19(3): 201-208.

Lunze, J. (2013). Regelungstechnik 2, 7th Edn., Springer, Berlin.

Lunze, J. (2017). Two methods to find analytical redundancy relations for fault diagnosis, Automatisierungstechnik 65(4): 219-232.

Massoumnia, M.-A., Verghese, G.C. and Willsky, A. (1989) Failure detection and identification, IEEE Transactions on Automatic Control 34(3): 316-321.

Murota, K. (1987). Systems Analysis by Graphs and Matroids, Springer, Berlin.

Pothen, A. and Fan, C.-J. (1990). Computing the block triangular form of a sparse matrix, ACM Transactions on Mathematical Software 16(4): 303-324.

Pröll, S., Jarmolowitz, F. and Lunze, J. (2015). Structural diagnosability analysis of switched systems, 9th IFAC Symposium on Fault Detection, Supervision and Safety of Technical Processes, Paris, France, pp. 156-163.

Reinschke, K.J. (1988). Multivariable Control, Akademie-Verlag, Berlin.

Shields, R. and Pearson, J. (1976). Structural controllability of multi-input linear systems, IEEE Transactions on Automatic Control 21(2): 203-212.

Svärd, C. and Nyberg, M. (2010). Residual generators for fault diagnosis using computation sequences with mixed causality applied to automotive systems, IEEE Transactions on Systems, Man and Cybernetics A: Systems and Humans 40(6): 1310-1328. 


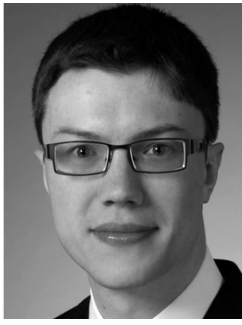

Sebastian Pröll was born in Ochsenfurt, Germany, in 1988. He received the MSc degree in mathematics from Julius-MaximiliansUniversity Würzburg in 2013. From 2013 to 2016 he was a researcher at Bosch Corporate Research. He currently works as a design engineer with the business sector at Chassis Systems Control of Robert Bosch GmbH. His research interests include model-based fault diagnosis using graph-theoretical methods and hybrid systems.

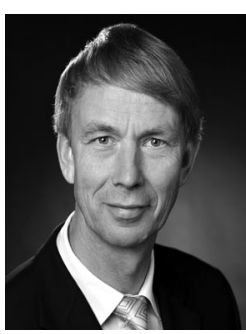

Jan Lunze is the head of the Institute of Automation and Computer Control at Ruhr University Bochum, Germany. His research interests include fault-tolerant control, networked control systems and hybrid dynamical systems. He is a co-author of the monograph Diagnosis and FaultTolerant Control, Springer, 2016 (3rd Edn.).

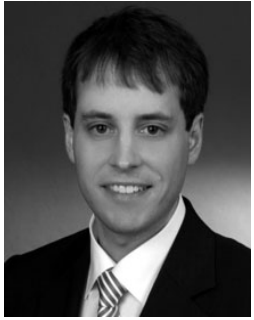

Fabian Jarmolowitz received his $\mathrm{PhD}$ degree in automatic control from RWTH Aachen University, Germany, in 2014. Right now he is a researcher at Corporate Research of Robert Bosch $\mathrm{GmbH}$. His research interests include fault diagnosis, model predictive control and uncertainty quantification.
Received: 13 March 2017

Revised: 24 October 2017

Accepted: 13 January 2018 